\title{
Walking through doorways causes forgetting: Environmental integration
}

\author{
Gabriel A. Radvansky, Andrea K. Tamplin, and Sabine A. Krawietz \\ University of Notre Dame, Notre Dame, Indiana
}

\begin{abstract}
Memory for objects declines when people move from one location to another (the location updating effect). However, it is unclear whether this is attributable to event model updating or to task demands. The focus here was on the degree of integration for probed-for information with the experienced environment. In prior research, the probes were verbal labels of visual objects. Experiment 1 assessed whether this was a consequence of an itemprobe mismatch, as with transfer-appropriate processing. Visual probes were used to better coordinate what was seen with the nature of the memory probe. In Experiment 2, people received additional word pairs to remember, which were less well integrated with the environment, to assess whether the probed-for information needed to be well integrated. The results showed location updating effects in both cases. These data are consistent with an event cognition view that mental updating of a dynamic event disrupts memory.
\end{abstract}

Previous work has shown that the architecture of the environment interacts with the architecture of cognition (Radvansky \& Copeland, 2006). Not only do people integrate contextual information into their memories, but how that context is structured and how it changes can also influence memory effectiveness. Specifically, when people move through doorways, memory for objects that have been interacted with is reduced. This has been interpreted from a situation model perspective (Zwaan \& Radvansky, 1998). However, because the term situation model is strongly associated with work in language comprehension, we use the term event model and extend these ideas to event cognition more broadly (Copeland, Magliano, \& Radvansky, 2006). The present study aimed to further test the generality of the principle that when there is an event boundary, thereby changing the event context, there is a disruption in the availability of information encountered as part of the prior event (Glenberg, Meyer, \& Lindem, 1987; Radvansky \& Copeland, 2010; Zwaan, 1996).

A direct assessment of the influence of changing events on memory was illustrated in a study by Radvansky and Copeland (2006), which is the basis for the present work. In this study, people moved through a multiroom virtual environment. Each room had one or two tables. A person first picked up an object (colored solids; e.g., a red cube or blue wedge) from a table and then moved to the next table. The person set the object down and picked up the next one. The person then moved to the table after that, and so on. At critical points, people were probed with the name of an object (e.g., red cube). These probes occurred either halfway across a large room (no-shift condition) or immediately after the person had entered a new room (shift condition). Following Glenberg et al. (1987), positive responses were to be made if the object was either the one currently being carried or the one just set down. Negative responses were for any other object. Negative probes were recombinations of object and color names from the two positive objects. So, if the object set down was a green pole and the carried object was a red cube, the negative probes could be either green cube or red pole.

When an object was picked up, it disappeared, so there was no visual reminder of what was being carried. Also, when moving, people turned their backs on the object that was just set down, so there was no visual reminder of it. Finally, memory probes did not occur at every possible location. This decreased the degree to which people anticipated being probed.

The results showed that people made more errors if they had moved to a new room. This is the location updating effect. This forgetting effect was supported by a response time difference, with people responding slower in the shift than in the no-shift condition. When people updated their event models, this compromised memory for the objects in the environment.

The event cognition explanation is that the location updating effect is a consequence of parsing a stream of events, thereby reducing the availability of information prior to an event shift (Kurby \& Zacks, 2008; Speer, Reynolds, Swallow, \& Zacks, 2009; Swallow, Zacks, \& Abrams, 2009; Zacks, Speer, \& Reynolds, 2009). This is not an effect of environmental context-dependent memory. Other work that we have done has found that reinstating the context does not improve performance. With location updating, a person modifies his or her event model to adjust to the

G. A. Radvansky, gabriel.a.radvansky.1@nd.edu 
new setting (Radvansky \& Copeland, 2010). Information that continues to be relevant across the two events is maintained, and information that was relevant to the prior event is removed. This event model updating should extend to a range of circumstances.

The present work explored the dependency of this effect on how well integrated the information in the memory probe is with the environmental context. It may vary with the degree of integration, where degree of integration refers to how much the probed-for information is part and parcel of the event in which a person is embedded. From a broader theoretical perspective, it is well known that context information is stored in memory traces (e.g., Smith \& Vela, 2001). However, instead of assessing the influence of context as a retrieval cue, we assessed the degree to which changes in context influence memory. One could argue that the degree to which information is tied to a particular context would modulate the influence of a change in context on later memory retrieval. Thus, the focus here is on the degree to which the probed-for information is embedded in the context of the structure of the ongoing event. In the present work, we explored this by using memory probes that were more (Experiment 1) or less (Experiment 2) tied to the experienced environment.

\section{EXPERIMENT 1}

In the Radvansky and Copeland (2006) study, although people saw images of objects, verbal probes were used. These probes were not well integrated with the experienced visual-spatial context. There was a mismatch of probe and target, such as that observed with transferappropriate processing (Kolers \& Roediger, 1984). Inconsistencies between how information is encoded and retrieved can hinder memory, opening a window to further disruptions, such as those produced by location updating. Similarly, in a study by Swallow et al. (2009), people were probed for objects seen in films: For objects that were relevant across event boundaries (as our probed-for objects were) and that were actually fixated during film viewing, Swallow et al. found poorer memory following an event shift when the task emphasized conceptual qualities (e.g., did you see a spatula or a pot?), but not when the task emphasized perceptual qualities (e.g., which of two pots did you see?). Thus, location updating may be disruptive when the probe task emphasizes conceptual qualities (e.g., using verbal labels of seen objects), but not when it emphasizes perceptual qualities (e.g., using pictures of objects).

Another view is that updating an event model following a location shift disrupts memory for information associated with a prior location (the objects in the previous room), even if it continues to be task relevant. How the information is probed for, whether verbal or visual, would be of minor consequence.

To test between these two accounts, in Experiment 1 half of the people received probes that were pictures of the objects (e.g., an image of a red cube rather than the words red cube). The rest received standard verbal probes. In addition, we altered how people navigated the environment. In prior work, people used arrow keys on a keyboard with their left hand, and they responded by pressing buttons on a mouse held in their right. In Experiment 1, people used a joystick to navigate and responded using two buttons on the joystick.

\section{Method}

Participants. Forty-nine people ( 28 female) from the University of Notre Dame were given partial course credit for their participation. Twenty-four were in the text-probe condition, and 25 were in the picture-probe condition.

Materials and Apparatus. The virtual spaces were created using the Valve Hammer program. This is the program used to create environments for the Half-Life video game. The displays were presented on a 66-in.-diagonal rear projection SmartBoard using a PC-compatible computer, with people seated about $1 \mathrm{~m}$ from the display, the room was darkened, and people wore headphones (so they could hear their "own footsteps"). The virtual space was a 53-room environment in which the rooms were of one of two sizes, with the large rooms being twice as long as the small rooms. This difference in room size allowed for the distance traveled to be equated in the shift and no-shift conditions, the only difference being whether a change in location occurred. In each room were one or two tables. Each table was placed along a wall in the room. In the small rooms there was only a single table, whereas in the large rooms there was a table on each half of the room. At one end of the table was the object the participant was to pick up. The other half of the table was empty. This is where the object being carried was to be put down. Finally, the two doorways in the room were never on the same wall.

The objects were made by combining colors and shapes. The colors used were red, orange, yellow, green, blue, purple, white, gray, brown, and black. The objects were all regular geometric shapes: cube, wedge, pole, disc, cross (X), and cone.

Procedure. After giving informed consent, people were seated in front of the display. Their task was to pick up an object from one room, go to the next room, place the object on the empty part of the table, pick up the next object, proceed to the next room, and so forth. When an object was picked up, it disappeared. Thus, a person could not see what he/she was currently carrying. When it was dropped off, it appeared on the table. Picking up and putting down objects was done by touching the appropriate end of the table.

To have people progress through the rooms in the required order, after they entered a room the door of entry closed. The door to the next room did not open until the person put down the carried object and picked up the new object. The doorway to the next room always required the person to turn away from the table in the current room.

There were 48 probe trials. Thus, not every possible test point had a probe. On probe trials, immediately upon entering a room or crossing halfway through a larger room, people were presented with a probe that appeared in the middle of the screen. This probe was either an image of an object or a color and shape name. People were to respond "yes" if the probe was either the object that was currently being carried or the one that had just been set down, and to respond "no" to all other probes. The negative probes were generated by recombining the object and color for these two objects. For example, if the carried object was a white cube, and the object set down was a red wedge, a negative probe might be red cube. Half of the probes occurred after a spatial shift, and half did not.

A joystick was held in the dominant hand for movement. For responding, a button pressed by the index finger (the trigger) was used for a positive response and a button on the top of the joystick was pressed with the thumb for a negative response. There were 24 positive probes and 24 negative probes. 
Table 1

Error Rates (Proportions) and Response Times (RTs, in Milliseconds), With Standard Errors, for Experiment 1

\begin{tabular}{ccccrr}
\hline & \multicolumn{2}{c}{ Error Rate } & & \multicolumn{2}{c}{ RT } \\
\cline { 2 - 3 } \cline { 5 - 6 } & $M$ & $S E$ & & $M$ & $S E$ \\
\hline Text Probes & & & & \\
$\quad$ No shift & .10 & .01 & & 1,317 & 79 \\
$\quad$ Shift & .16 & .03 & & 1,564 & 119 \\
Picture Probes & & & & \\
$\quad$ No shift & .15 & .03 & & 1,322 & 59 \\
Shift & .20 & .04 & & 1,761 & 138 \\
\hline
\end{tabular}

\section{Results}

The error rate and response time data are reported in Table 1 and were submitted to 2 (probe type: text vs. picture probes) $\times 2$ (shift condition: no-shift vs. shift) mixed ANOVAs, with the first factor between subjects and the other factor within subjects. For the error rates, the main effect of probe type was not significant $[F(1,46)=2.12$, $\left.M S_{\mathrm{e}}=.022, p=.15\right]$, but the effect of shift was $[F(1,46)=$ $\left.4.26, M S_{\mathrm{e}}=.035, p=.05\right]$, with people making more errors when there was a shift than when there was not. The interaction was not significant $(F \mathrm{~s}<1)$. Thus, there was forgetting after moving from one location to another, but the nature of the probe (picture or verbal) had no meaningful effect.

For the response time analysis, errors were excluded, and we trimmed the data by removing response times faster than $200 \mathrm{msec}$ and slower than 10,000 msec. Then the data were submitted to the van Selst and Jolicœur (1994) trimming procedure, which is based on the number of observations per cell, with $6 \%$ of the data being dropped. Again, while the main effect of probe type was not significant $(F<1)$, the effect of shift was $[F(1,46)=$ $\left.21.15, M S_{\mathrm{e}}=133,469, p=.001\right]$, with people responding slower following the shift as opposed to the no-shift conditions. The interaction was not significant $[F(1,46)=$ $\left.1.67, M S_{\mathrm{e}}=133,469, p=.20\right]$.

\section{Discussion}

Experiment 1 assessed whether the location updating effect was due to poorer integration of the memory probes with event context because of their verbal nature. However, this effect was present for both visual and verbal probes. This is consistent with the view that when participants move from one location to another, information that was relevant in the prior location becomes generally less available. People are creating event models of the ongoing circumstances, and updating these models can make some information less available. The apparent discrepancy between our findings and those of Swallow et al. (2009) regarding conceptual versus perceptual processing is likely due to methodological differences. For example, they manipulated the nature of the memory task, whereas we always used recognition. Moreover, objects were the focus of our task but were often incidental in the film scenes in the Swallow et al. work. Finally, having people use a joystick to navigate the environment had no discernible influence on performance.

\section{EXPERIMENT 2}

Experiment 2 assessed the availability of information that is less well integrated with the environment. Specifically, in addition to memory for the objects, we assessed memory for word pairs that did not refer to features of the context in which they were encountered. They were random word pairs that were presented simply to be remembered. When a probe was presented, it could be either an object name or a word pair.

Object probes were the same as in Experiment 1. For the word pairs, we used adjective-noun pairs (e.g., ethniccake) to parallel the structure of the object names (e.g., white wedge). These word pairs were nonsensical, further separating them from the environment. Moreover, adjectives and nouns were repeated, in different combinations, over the course of the trials, just like the object color and shape names. This also allowed for some proactive interference, making the task more demanding.

One possibility was that, because the word pairs were not contextually well integrated, they would be loosely associated with the event model and would be isolated from the effects of location updating. As such, there would be a location updating effect for the objects, but not word pairs. In contrast, an alternative view would be that the wide range of information processed as part of the ongoing situation would be integrated into the event model (e.g., Swallow et al., 2009), even if it was not well integrated with the external event. Thus, location updating would compromise memory for the word pairs as well as the objects.

\section{Method}

Participants. Forty people (20 female) from the University of Notre Dame were given partial course credit for participation. The data from two additional people were dropped because their error rates were close to chance.

Materials and Procedure. The same apparatus and a similar set of materials and procedures were used as in Experiment 1, with some important differences. First, the virtual space was a 74-room environment. Second, to parallel the color-shape names, the word pairs were adjective-noun combinations. These words were matched to the color and object names for number of letters, syllables, and word frequency, although the combinations were less meaningful. The adjectives used were marine, ethnic, third, chief, meet, north, dank, large, select, six, and fast. The nouns were cake, cuff, feat, lamp, wharf, $Q$, and $V$ (two letters were used because the shapes were referred to by letters; i.e., $T$ and $X$ ). The adjectives and nouns were repeated across trials just as the object color and shape names. Negative word probes were recombinations of adjectives and nouns from the two most recently seen word pairs. Prior to picking up each object, a word pair was displayed for $2 \mathrm{sec}$. The task was to hold it in memory in case it was probed for at one of the probe locations. Arrow keys on the keyboard and the mouse buttons were used to move and respond to probes, respectively.

For the critical trials, upon entering a room or crossing the halfway point of a large room, people were presented with a probe that could be either an object or a word pair. When the probe was an object, the task was the same as in Experiment 1. In comparison, when it was a word pair, the person indicated whether it was the tobe-remembered word pair. There were 48 probe trials.

There were 24 positive and 24 negative object probes. For the word pair probes, there were 12 positive and 12 negative probes. Half of the probes in each condition occurred after a location shift, and half did not. 
Table 2

Error Rates (Proportions) and Response Times (RTs, in Milliseconds), With Standard Errors, for Experiment 2

\begin{tabular}{llllrr}
\hline & \multicolumn{3}{c}{ Error Rate } & & \multicolumn{2}{c}{ RT } \\
\cline { 2 - 3 } \cline { 5 - 5 } & $M$ & $S E$ & & $M$ & $S E$ \\
\hline Object Probes & & & & \\
$\quad$ No shift & .18 & .02 & & 1,747 & 82 \\
$\quad$ Shift & .23 & .03 & & 1,978 & 101 \\
$\quad$ Word Pair Probes & & & & \\
$\quad$ No shift & .08 & .02 & 1,234 & 45 \\
$\quad$ Shift & .14 & .02 & 1,306 & 64 \\
\hline
\end{tabular}

\section{Results}

The error rate and response time data are summarized in Table 2. The object and word probe data were analyzed separately. The response time data were trimmed as in Experiment 1 , with $8 \%$ of the data being dropped. The data were submitted to two-way (shift vs. no-shift) repeated measures ANOVAs.

Object probes. For the error rates, the location updating effect was significant $\left[F(1,39)=4.29, M S_{\mathrm{e}}=.012\right.$, $p=.04]$. Walking through a doorway caused forgetting of the objects. This was supported by the response time data $\left[F(1,39)=12.31, M S_{\mathrm{e}}=87,164, p=.001\right]$.

Word pair probes. There was a location updating effect for the error rate data $\left[F(1,39)=5.47, M S_{\mathrm{e}}=.016\right.$, $p=.03]$, with people forgetting the word pair more often when they needed to update the event model. This difference, although in the appropriate direction, did not reach significance in the response times $\left[F(1,39)=2.18, M S_{\mathrm{e}}=\right.$ $48,029, p=.15]$.

\section{Discussion}

The results of Experiment 2 showed a location updating effect for both objects and word pairs. These data are more consistent with the view that most of what a person is attending to becomes less available following an event boundary as the event model is updated.

\section{GENERAL DISCUSSION}

Two experiments further explored the location updating effect (Radvansky \& Copeland, 2006), in which people show poorer memory for objects after a shift from one room to another. This effect was not limited by how strongly the probed-for information was integrated with the context of the interactive environment. People in Experiment 1 showed that using visual probes that were a better match for that information from the environmental context did not add any benefit to retrieval. In Experiment 2, word pairs that were less well integrated also showed the effect. Thus, both increasing and decreasing the degree of integration of the probed-for information consistently resulted in a location updating effect.

Moving from one location to another serves as an event boundary (e.g., Swallow et al., 2009) as information associated with a prior location becomes less available. This is noteworthy in that in the present study, (1) the amount of to-be-remembered information was relatively small and (2) the task demanded that information was relevant and needed to be maintained. Thus, the event shift had a broad-based impact on memory. These data are in line with other event cognition findings in which information was affected by the process of updating the changing event model.

The present research is also consistent with a range of findings in spatial cognition that show the importance of spatial regions. For example, spatial regions can be used to integrate and segregate information in long-term memory, thereby influencing the pattern of retrieval interference (e.g., Radvansky, 1999, 2005; Radvansky, Spieler, \& Zacks, 1993; Radvansky \& Zacks, 1991) and the retrieval of information from a mental map (e.g., Bower \& Rinck, 2001; McNamara, 1986; Thorndyke, 1981). In research on narrative comprehension, memory for objects declines when there has been a shift in location (e.g., Curiel \& Radvansky, 2002; Glenberg et al., 1987; Morrow, Greenspan, \& Bower, 1987; Radvansky \& Copeland, 2010; Rinck \& Bower, 1995). Also, people read more slowly when they encounter a spatial shift in a text (Zwaan, Magliano, \& Graesser, 1995; Zwaan, Radvansky, Hilliard, \& Curiel, 1998) and organize narrative information by spatial regions (Radvansky, 1999; Radvansky et al., 1993; Radvansky \& Zacks, 1991; Zwaan, Langston, \& Graesser, 1995), even with more perceptual events, such as narrative film (Magliano, Miller, \& Zwaan, 2001).

In sum, walking through doorways reduces the availability of information in memory. This is relatively unaffected by the degree of integration of the information with the surrounding environmental context. Movement from one location to another disrupts cognition. Ongoing and future research is aimed at understanding the underlying causes of this forgetting.

\section{AUTHOR NOTE}

We thank Daniel Blakely, Mark Bohay, Abbi Daugherty, Erica Nason, Patrick O'Keefe, Jenny Walls, Megan Cefferillo, and Brittany Gragg for their assistance in collecting the data. We also thank Jeff Smith and Mike Villano for their programming expertise. This research was supported in part by a grant from the Army Research Institute, ARMY-DASW01-02-K-0003, and by funding from J. Chris Forsythe of Sandia National Laboratories. Please address correspondence to G. A. Radvansky, Department of Psychology, University of Notre Dame, Notre Dame, IN 46556 (e-mail: gabriel.a.radvansky.1@nd.edu).

Note-Accepted by Cathleen M. Moore's editorial team.

\section{REFERENCES}

Bower, G. H., \& RincK, M. (2001). Selecting one among many referents in spatial situation models. Journal of Experimental Psychology: Learning, Memory, \& Cognition, 27, 81-98. doi:10.1037/0278 $-7393.27 .1 .81$

Copeland, D. E., Magliano, J. P., \& Radvansky, G. A. (2006). Situation models in comprehension, memory, and augmented cognition. In C. Forsythe, M. L. Bernard, \& T. E. Goldsmith (Eds.), Cognitive systems: Human cognitive models in systems design (pp. 37-66). Mahwah, NJ: Erlbaum.

Curiel, J. M., \& Radvansky, G. A. (2002). Mental maps in memory retrieval and comprehension. Memory, 10, 113-126. doi:10.1080/ 09658210143000245

Glenberg, A. M., Meyer, M., \& Lindem, K. (1987). Mental models contribute to foregrounding during text comprehension. Journal of Memory \& Language, 26, 69-83. doi:10.1016/0749-596X(87)90063-5

Kolers, P. A., \& Roediger, H. L., III (1984). Procedures of mind. Jour- 
nal of Verbal Learning \& Verbal Behavior, 23, 425-449. doi:10.1016/ S0022-5371(84)90282-2

Kurby, C. A., \& ZaCKs, J. M. (2008). Segmentation in the perception and memory of events. Trends in Cognitive Sciences, 12, 72-79. doi:10.1016/j.tics.2007.11.004

Magliano, J. P., Miller, J., \& ZwaAn, R. A. (2001). Indexing space and time in film understanding. Applied Cognitive Psychology, 15, 533-545. doi:10.1002/acp.724

McNamara, T. P. (1986). Mental representations of spatial relations. Cognitive Psychology, 18, 87-121. doi:10.1016/0010-0285(86)90016-2

Morrow, D. G., Greenspan, S. L., \& Bower, G. H. (1987). Accessibility and situation models in narrative comprehension. Journal of Memory \& Language, 26, 165-187. doi:10.1016/0749-596X(87)90122-7

Radvansky, G. A. (1999). The fan effect: A tale of two theories. Journal of Experimental Psychology: General, 128, 198-206. doi:10.1037/ 0096-3445.128.2.198

Radvansky, G. A. (2005). Situation models, propositions, and the fan effect. Psychonomic Bulletin \& Review, 12, 478-483.

Radvansky, G. A., \& Copeland, D. E. (2006). Walking through doorways causes forgetting: Situation models and experienced space. Memory \& Cognition, 34, 1150-1156.

Radvansky, G. A., \& Copeland, D. E. (2010). Reading times and the detection of event shift processing. Journal of Experimental Psychology: Learning, Memory, \& Cognition, 36, 210-216. doi:10.1037/ a0017258

Radvansky, G. A., Spieler, D. H., \& Zacks, R. T. (1993). Mental model organization. Journal of Experimental Psychology: Learning, Memory, \& Cognition, 19, 95-114. doi:10.1037/0278-7393.19.1.95

Radvansky, G. A., \& Zacks, R. T. (1991). Mental models and the fan effect. Journal of Experimental Psychology: Learning, Memory, \& Cognition, 17, 940-953. doi:10.1037/0278-7393.17.5.940

RiNCK, M., \& BowER, G. H. (1995). Anaphora resolution and the focus of attention in situation models. Journal of Memory \& Language, 34, 110-131. doi:10.1006/jmla.1995.1006

Smith, S. M., \& Vela, E. (2001). Environmental context-dependent memory: A review and meta-analysis. Psychonomic Bulletin \& Review, 8, 203-220.
Speer, N. K., Reynolds, J. R., Swallow, K. M., \& Zacks, J. M. (2009). Reading stories activates neural representations of visual and motor experiences. Psychological Science, 20, 989-999. doi:10.1111/j.1467 $-9280.2009 .02397 . x$

Swallow, K. M., ZaCKs, J. M., \& Abrams, R. A. (2009). Event boundaries in perception affect memory encoding and updating. Journal of Experimental Psychology: General, 138, 236-257. doi:10.1037/ $\mathrm{a} 0015631$

Thorndyke, P. W. (1981). Distance estimation from cognitive maps. Cognitive Psychology, 13, 526-550. doi:10.1016/0010-0285(81)90019-0

VAN SElst, M., \& Jolicceur, P. (1994). A solution to the effect of sample size on outlier elimination. Quarterly Journal of Experimental Psychology, 47A, 631-650

ZACKs, J. M., SpeEr, N. K., \& Reynolds, J. R. (2009). Segmentation in reading and film comprehension. Journal of Experimental Psychology: General, 138, 307-327. doi:10.1037/a0015305

ZwAAN, R. A. (1996). Processing narrative time shifts. Journal of Experimental Psychology: Learning, Memory, \& Cognition, 22, 11961207. doi:10.1037/0278-7393.22.5.1196

Zwaan, R. A., Langston, M. C., \& Graesser, A. C. (1995). The construction of situation models in narrative comprehension: An eventindexing model. Psychological Science, 6, 292-297. doi:10.1111/ j.1467-9280.1995.tb00513.x

Zwann, R. A., Magliano, J. P., \& Graesser, A. C. (1995). Dimensions of situation model construction in narrative comprehension. Journal of Experimental Psychology: Learning, Memory, \& Cognition, 21, 386-397. doi:10.1037/0278-7393.21.2.386

ZWAan, R. A., \& Radvansky, G. A. (1998). Situation models in language comprehension and memory. Psychological Bulletin, 123, 162185. doi:10.1037/0033-2909.123.2.162

Zwaan, R. A., Radvansky, G. A., Hilliard, A. E., \& Curiel, J. M. (1998). Constructing multidimensional situation models during reading. Scientific Studies of Reading, 2, 199-220. doi:10.1207/ s1532799xssr0203_2

(Manuscript received February 11, 2010; revision accepted for publication July 17, 2010.) 